\title{
Le commerce du blé et la navigation dans le Bas-Richelieu avant 1849
}

\section{P.-André Sévigny}

Volume 38, numéro 1, été 1984

URI : https://id.erudit.org/iderudit/304234ar

DOI : https://doi.org/10.7202/304234ar

Aller au sommaire du numéro

Éditeur(s)

Institut d'histoire de l'Amérique française

ISSN

0035-2357 (imprimé)

1492-1383 (numérique)

Découvrir la revue

Citer cet article

Sévigny, P.-A. (1984). Le commerce du blé et la navigation dans le Bas-Richelieu avant 1849. Revue d'histoire de l'Amérique française, 38(1), 5-21.

https://doi.org/10.7202/304234ar d'utilisation que vous pouvez consulter en ligne.

https://apropos.erudit.org/fr/usagers/politique-dutilisation/ 


\title{
LE COMMERCE DU BLÉ ET LA NAVIGATION DANS LE BAS-RICHELIEU AVANT 1849*
}

\author{
P.-ANDRÉ SÉVIGNY \\ Parcs Canada \\ Québec
}

\begin{abstract}
À la suite d'études historiques consacrées au canal Chambly ${ }^{1}$ et à l'écluse Saint-Ours ${ }^{2}$, des structures mises en service sur la rivière Richelieu en 1843 et 1849 respectivement, nous nous sommes intéressé à la navigation commerciale le long de cette voie d'eau avant l'entrée en scène de ces auxiliaires technologiques. Deux phénomènes retinrent particulièrement notre attention: la prépondérance du blé dans ce transport mais aussi les mauvaises conditions de circulation sur le bas Richelieu ${ }^{3}$ entre Sorel et Chambly.

Par ailleurs, en abordant cette question des céréales, nous avons trouvé, en toile de fond, le débat historiographique entourant la «crise agricole» qui affligea, semble-t-il, le Bas-Canada vers 1830. Crise structurelle attribuable à une chute de la production et de la productivité causée par l'épuisement des sols ${ }^{4}$ ? Crise conjoncturelle reliée à l'accroissement de la population, à la raréfaction des terres et à la diversification des marchés ${ }^{5}$ ?

Les pages qui suivent n'ont certes pas l'intention de relancer ou d'aviver ce débat. Elles veulent seulement illustrer certaines modalités
\end{abstract}

* Historien à Parcs Canada, région du Québec, l'auteur étứdie depuis quelques années diverses questions entourant la canalisation de la rivière Richelieu. À l'instar de plusieurs autres canaux de navigation canadiens, le canal Chambly et l'écluse Saint-Ours ont été confiés à Parcs Canada en 1972 à titre de parcs historiques nationaux.

1 P.-André Sévigny, Commerce et navigation sur le canal Chambly: aperçu historique, Études en archéologie, architecture et histoire (Parcs Canada, Environnement Canada, Ottawa, 1983).

P.-André Sévigny, Navigation et canalisation dans le Bas-Richelieu au milieu du dixneuvième siècle: l'écluse et le barrage Saint-Ours, rapport interne de recherche (Parcs Canada, Québec, 1983).

${ }_{3}$ La Commission de toponymie du Québec recommande d'écrire «bas Richelieu» lorsque nous parlons du cours d'eau proprement dit et «Bas-Richelieu» si nous faisons référence à la région géographique ou au bassin hydrographique de la rivière.

${ }_{4}$ Les principaux défenseurs de cette thèse sont Fernand Ouellet et Jean Hamelin. Ils ont bien exposé leur point de vue dans «Les rendements agricoles dans les seigneuries et les cantons du Québec, 1760-1850», dans C. Galarneau et E. Lavoie, éd., France et Canada français du XVI ${ }^{e}$ au XX $X^{e}$ siècle (Québec, 1963), 81-120.

5 Gilles Paquet et Jean-Pierre Wallot, «Crise agricole et tensions socio-ethniques dans le Bas-Canada, 1802-1812: éléments pour une interprétation», Revue d'histoire de l'Amérique française, 26, 2 (septembre 1972): 203, 221. Le géographe Serge Courville va même jusqu'à affirmer que «rien dans la dialectique spatiale de la thèse classique ne permet de soutenir l'existence d'une crise agricole dans le Bas-Canada.» «La crise agricole du Bas-Canada: éléments d'une réflexion géographique», Cahiers de géographie du Québec, 24, 62 (septembre 1980): 221. 


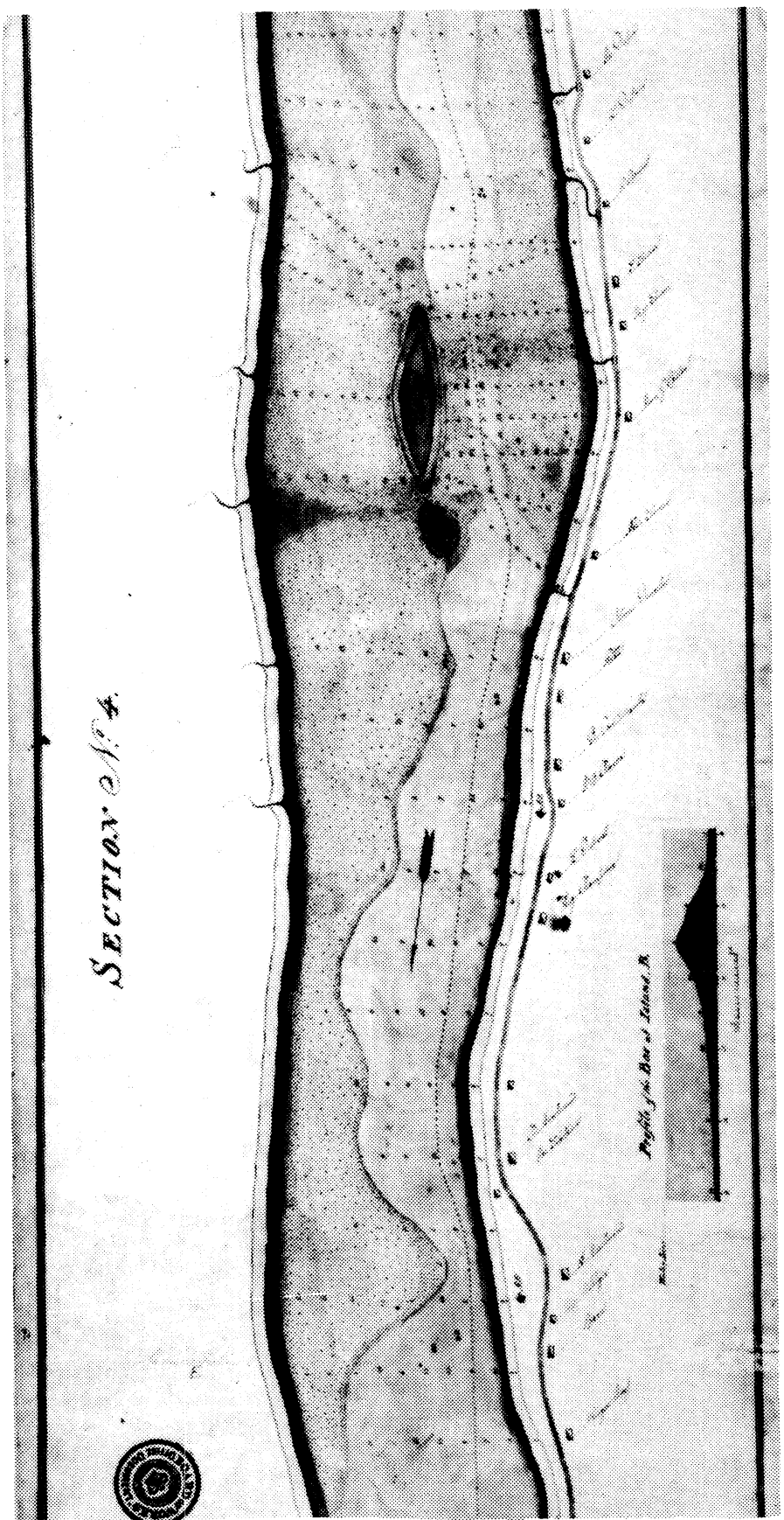

Les obstacles à la navigation sur le Bas-Richelieu en 1830. Suite à une inspection de la région, Augustus Kuper a tracé la carte des trois secteurs les plus difficiles de la rivière. Les trois photographies reproduites ici sont celles du secteur Saint-Denis/ Saint-Antoine.

Source-crédit: Archives publiques du Canada, Collection nationale de cartes et plans H1/310 - Richelieu 1830, NMC-13197, 13198, 13199. 


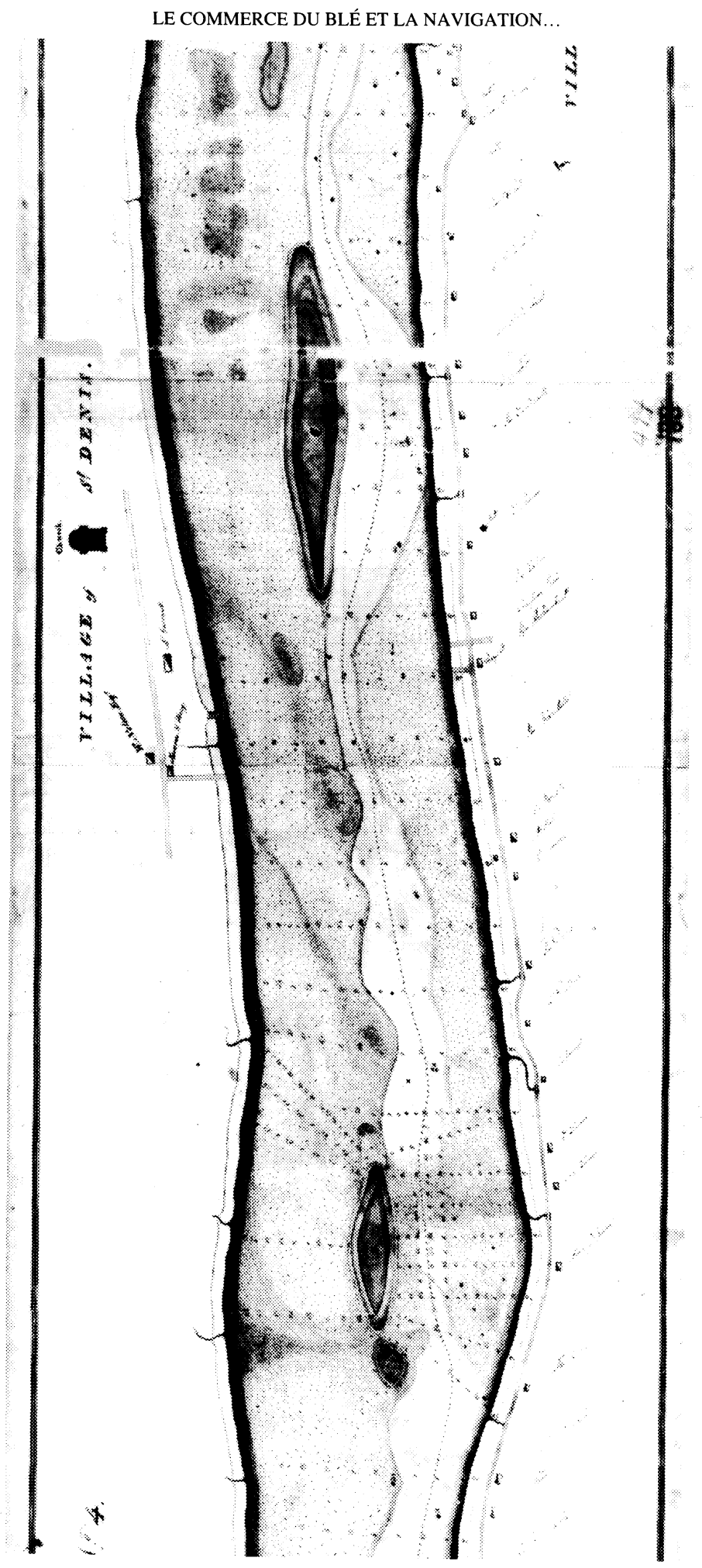




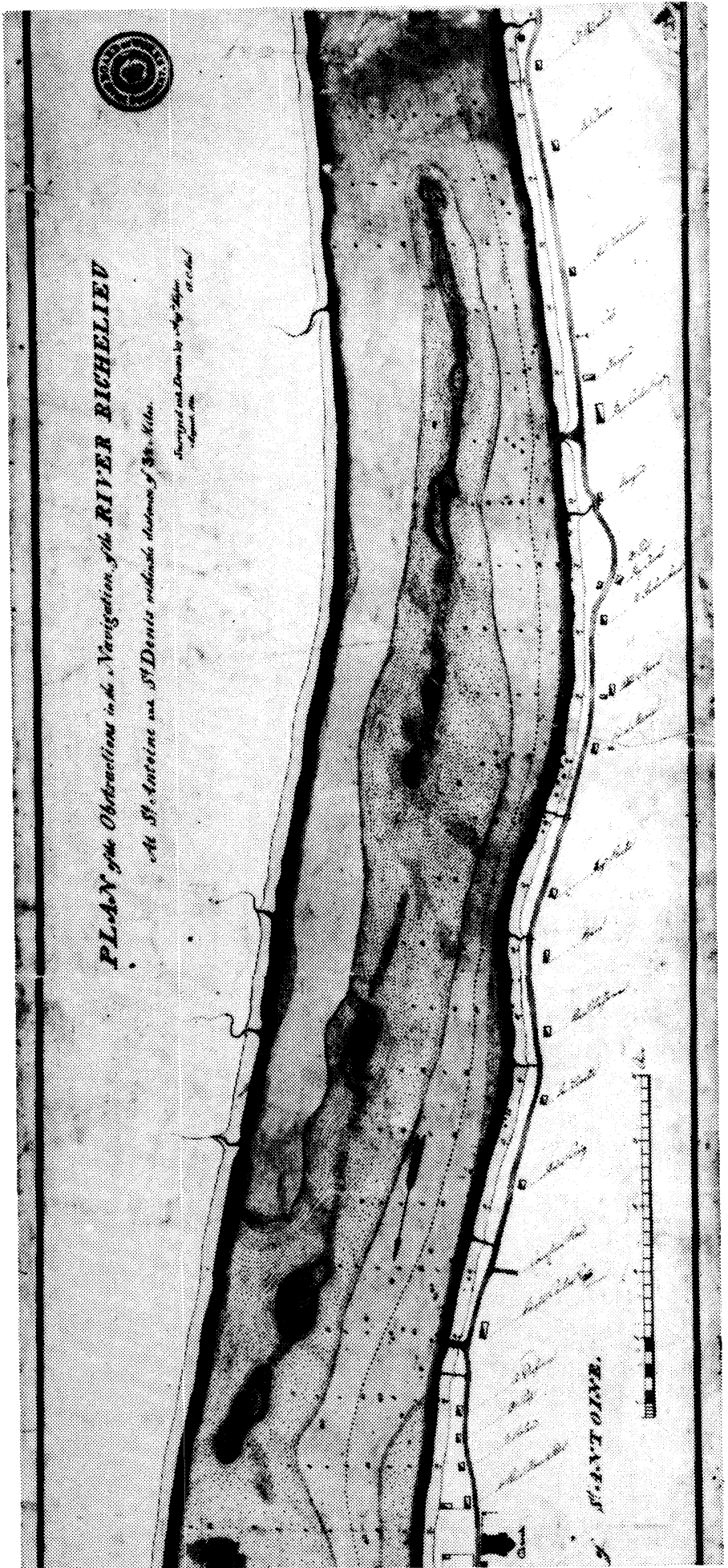


du commerce des grains dans ce grenier du Bas-Canada qu'était alors la basse vallée de la rivière Richelieu.

\section{Quelques figures de négociants}

C'est un fait bien connu que l'économie du Bas-Richelieu des années 1760-1860 reposait sur la production de grains, le blé particulièrement, mais aussi l'avoine, l'orge, le seigle, les pois. Voilà ce que la vallée était en mesure d'exporter: une véritable manne pour toute la colonie laurentienne et même pour l'étranger, surtout durant les années 1790-1815. Les promoteurs locaux ou régionaux de ce négoce lucratif furent assez nombreux, au fil des décennies, à marchander les grains de la vallée; mais certains noms méritent davantage d'être retenus, surtout en raison de l'ampleur de leurs opérations.

Samuel Jacobs arriva au Canada au lendemain de la Conquête à titre, semble-t-il, de pourvoyeur des troupes anglaises. Ce marchand juif se hâta d'établir des magasins d'approvisionnement militaire depuis Crown Point, état de New York, jusqu'à Sorel. Il brassa de nombreuses affaires avec les fermiers de Saint-Ours et de Saint-Charles, mais c'est à Saint-Denis qu'il établit sa résidence, là où dès 1763 , il avait ouvert une sorte de magasin général. Il achetait les grains de la région, exploitait une distillerie et exportait de la perlasse en Angleterre ${ }^{6}$.

Jacobs, qui mourut en 1786, était à la fois grossiste et détaillant, appuyé financièrement sur le marché des grains, mais impliqué dans le commerce de plusieurs autres produits, partagé en affaires entre la ville et la campagne, entre les produits de consommation courante et le blé. Il fut sans doute également l'un des premiers expéditeurs de la région, sous le régime britannique, à se mesurer au Richelieu, à en comprendre le mode d'utilisation. Il faut savoir en effet que, pour acheminer ses grains vers le Saint-Laurent, le marchand devait s'y prendre tôt au printemps, alors que de petites goélettes, d'une capacité de 1000 à 1500 minots, étaient en mesure d'éviter les rapides et hauts-fonds en bas de Saint-Denis. Â partir du mois de juin, c'est à Saint-Ours qu'il fallait d'abord acheminer le grain, le plus souvent dans des barques peu spacieuses et à fond plat; à cet endroit, des bateaux d'une capacité de 2000 minots pouvaient accoster et être chargés durant toute la saison de navigation ${ }^{7}$.

À Saint-Antoine, juste en face de Saint-Denis, était établie à la même époque la famille Cartier. Durant trois générations, les Cartier s'illustrèrent en tant que marchands céréaliers. Au cours de la période

\footnotetext{
6 Denis Vaugeois, «Samuel Jacobs», Dictionnaire biographique du Canada (Québec, 1980), IV: $415-417$.

7 Allan Greer, «Habitants of the Lower Richelieu: Rural Society in Three Quebec Parishes, 1740-1840», thèse de doctorat, York University, Toronto (1980), 283.
} 
1770-1840, ils auraient même dominé cet important secteur commercial dans le Bas-Canada ${ }^{8}$. Examinons donc les activités de cette petite dynastie, surtout connue dans l'histoire pour avoir donné le jour à un homme politique célèbre, George-Étienne Cartier (1814-1898).

L'ancêtre, Jacques Cartier I (1720-1770), arriva au Canada vers 1735-1740, en provenance de l'Anjou. Marié à Beauport à Marguerite Mongeon en 1744, il aurait été, dès 1750, un important marchand de sel et de poisson de Québec. Après la Conquête, il augmenta sa fortune en approvisionnant l'armée anglaise, à la façon de Samuel Jacobs ${ }^{9}$. On dit qu'il aurait abordé le marché du blé et de la potasse pour profiter de la demande des nouveaux dirigeants pour ces denrées; il envoya ses deux fils, Jacques II (1750-1814) et Joseph (1752-1815), prospecter la vallée du Richelieu, une région déjà fort connue pour sa fertilité et sa productivité. Les deux garçons décidèrent rapidement de s'y établir et de devenir les représentants de leur père dans le négoce des grains et des marchandises en gros. Jacques II s'installa à Saint-Antoine, pendant que Joseph construisait sa demeure de l'autre côté de la rivière, à Saint-Denis. C'est d'ailleurs à cet endroit qu'ils érigèrent leurs premiers quais et entrepôts.

Lorsque l'ancêtre mourut, en 1770, il laissait une entreprise florissante. Des milliers de boisseaux de blé étaient expédiés annuellement de la vallée du Richelieu en bateaux et parvenaient à Québec où ils étaient moulus dans les deux gros moulins à farine appartenant à la famille. Deux énormes entrepôts de pierre emmagasinaient en outre les grains le long des quais de la capitale. Au début du dix-neuvième siècle, l'un des deux frères, Joseph, se lança à son compte et s'installa à SaintHyacinthe. Son fils, Joseph II, le remplaça comme associé de Jacques II.

En 1806, les Cartier se départirent de la section québécoise de leur entreprise; les moulins à farine de l'ancêtre furent vendus. De mauvaises récoltes, l'inflation et la compétition américaine avaient fait chuter les exportations de blé vers l'Angleterre: un million de boisseaux avaient quitté le port de Québec en 1802; trois ans plus tard, en 1805, ces envois ne se chiffraient plus qu'à 22000 boisseaux. Les Cartier allaient donc concentrer leurs efforts dans la vallée du Richelieu, à proximité de Montréal, cet important marché étant en pleine croissance ${ }^{10}$. Quant à leurs exportations de blé outre-mer, elles seraient

$8 \quad$ Alastair Sweeny, George-Étienne Cartier, A Biography (Toronto, 1976), 19.

9 C'est essentiellement par le biais des biographies de George-Étienne Cartier que nous connaissons la famille Cartier, ancêtre et descendants. Voir particulièrement les ouvrages suivants: A. Sweeny, op . cit., 18-24; Brian Young, George-Étienne Cartier, Montréal Bourgeois (Kingston et Montréal, 1981), 1-2; Henry M. Best, «George-Étienne Cartier», thèse de doctorat, Université Laval, Québec (1969), 11.

10 A. Sweeny, op cit., 24. 
dorénavant confiées aux courtiers québécois George Symes et Austin Cuvillier.

Outre Samuel Jacobs et la famille Cartier, il se trouvait quelques autres négociants d'importance dans les paroisses du Bas-Richelieu au cours de la première moitié du dix-neuvième siècle. À Saint-Mathias, par exemple, nous pouvions rencontrer Timothée Franchère et Eustache Soupras, commerçants, capitaines de milice et défenseurs très actifs des projets de canalisation du Richelieu.

À la tête de deux sociétés commerciales distinctes, ces deux éminences régionales opéraient à partir de la Pointe-aux-Oliviers (SaintMathias) où étaient érigés leurs principaux magasins et entrepôts. Depuis la guerre de 1812, particulièrement, leur compagnie respective s'était développée à un rythme accéléré. L'apparition des bateaux à vapeur y était certes pour quelque chose. Franchère, outre son poste de SaintMathias, comptait sur des entrepôts disséminés à travers la région, une petite flotte de goélettes, un important magasin à Sainte-Marie de Monnoir, sur la rivière Yamaska, et une succursale à Saint-Athanase, dans la seigneurie de Bleury. Quant à Soupras, depuis Saint-Mathias il rayonnait surtout en direction de Saint-Jean-Baptiste de Rouville et de la rivière Yamaska ${ }^{11}$.

Parmi les plus importants marchands céréaliers du Richelieu, il faut mentionner enfin le nom de Pierre Guérout, installé solidement à Saint-Denis. Né en Allemagne en 1753, il vint au pays vers 1780 avec les troupes levées outre-Rhin par l'Angleterre. Licencié, il s'établit à Saint-Antoine en qualité de marchand mais, en 1786, il traversa à SaintDenis.

Il accrut alors de beaucoup son commerce. Son comptoir primitif devint un véritable entrepôt où les autres négociants venaient même s'approvisionner de loin. En retour, il remplissait d'immenses hangars de toutes sortes de grains, surtout du blé. Aux eaux hautes du printemps, il avait sa barge spéciale qui montait de Québec pour ravitailler le magasin et charger les céréales. ${ }^{12}$

Pierre Guérout fit rapidement fortune. Il décéda vers 1830 et son fils Louis lui succéda à la tête de l'entreprise.

Les hommes d'affaires de cette taille n'étaient pas nombreux dans la région. Remarquons, à cet égard, qu'aucun d'entre eux n'habite SaintOurs; en effet, Saint-Mathias, Saint-Denis et Saint-Antoine sont les villages qui, depuis la Conquête, attirent les plus importants commerçants de grain. Nous disons «attirent», car, à l'instar d'Allan Greer,

\footnotetext{
11 Rosaire Benoît, «Une école de syndics à Saint-Mathias en 1832», Les cahiers de la seigneurie de Chambly, IV, 2 (1982): 11-12.

${ }_{12}$ Jean-Baptiste-Arthur Allaire, Histoire de la paroisse de Saint-Denis-sur-Richelieu (SaintHyacinthe, 1905), 211.
} 
nous constatons que si la basse vallée compte de nombreux marchands dans les décennies qui suivent les années 1760 , la plupart d'entre eux ne sont pas originaires de la région et y sont installés d'assez fraîche date $^{13}$. C'est le cas des Jacobs, Cartier et Guérout, mais aussi de Charles Curtius, John Grant, Marin Jehanne et autres.

\section{Grossistes et détaillants}

L'absence de marchands céréaliers de grande envergure à SaintOurs étonne, considérant la fertilité de l'arrière-pays et surtout la localisation stratégique du village par rapport à la rivière. Bien sûr les Louis Marchand, Amable Arpin, Jean Bistodeau et Hyppolite Mogé ne sont pas des inconnus en aval de Chambly ${ }^{14}$. Nous retrouvons ici et là leur trace dans la documentation commerciale de l'époque mais il saute aux yeux qu'ils ne brassent pas des affaires aussi importantes que leurs homologues de Saint-Denis, Saint-Antoine ou Saint-Mathias. Ces cultivateurs, ou fils de cultivateurs recyclés, disposent-ils d'un capital suffisant pour mettre sur pied une entreprise commerciale vraiment importante? Chose certaine, ils ne jouissent pas dans les grands centres de «l'organisation» dont sont dotés les plus grands: courtiers, entrepôts, petite flotte, etc.

Ce qui différencie les petits et les gros commerçants, c'est en définitive la capacité des derniers de se hisser au-dessus du simple commerce de détail. Samuel Jacobs, par exemple, ne se contentait pas des entrées de blé provenant de son magasin de Saint-Denis; de petits marchands ruraux à Chambly, Beloeil, Varennes et ailleurs dans la région, il achetait en gros de grandes quantités de grains, pour son propre compte ou pour des correspondants québécois. C'est en général à son courtier de la capitale, John Welles, puis Charles Grant, qu'il vendait ses céréales; ceux-ci, en retour, lui fournissaient rhum, sel, étoffes et articles divers pour son commerce de détail. En plus de surveiller les intérêts de Jacobs, Welles et Grant lui procuraient du crédit, au besoin, et, plus important encore, l'informaient des prix et des fluctuations du marché à Québec ${ }^{15}$.

Somme toute, ce qui caractérise essentiellement Samuel Jacobs, Pierre Guérout ou les Cartier, c'est leur intérêt pour les grains, et spécialement le blé, en ces années où cette denrée constitue un pilier de l'économie nationale. La quête du blé devient vite une course effrénée, d'autant plus que la compétition est grande; les exportateurs de Québec envoient souvent leurs agents dans toutes les campagnes pour transiger directement avec les habitants, coupant ainsi l'herbe sous le pied des

13 Allan Greer, op. cit., 281.

14 A. Couillard-De:sprés, Histoire de la famille et de la seigneurie de Saint-Ours (Montréal, 1915 et 1917,2 vol.), II: 386-387.

15 A. Greer, op. cit., 282-283. 
marchands régionaux. Ces «traficeurs d'en bas», comme les appelle Wolfred Nelson ${ }^{16}$, font du porte à porte, offrant des prix élevés aux cultivateurs, sans parler des avances souvent consenties et même des bonus en argent ou en rhum. Inutile de dire que les expéditeurs richelois n'ont d'autre choix que d'offrir des conditions similaires ${ }^{17}$.

Le commerce de détail, bien sûr, ne rapportait pas autant mais il n'avait pas non plus à supporter une telle concurrence. En 1833, J.-M. Spink ouvre un nouveau magasin général à Saint-Charles-sur-Richelieu, bourg alors connu sous le nom de «village Debartzch». Il a pignon sur rue à un endroit stratégique, "près du quai du bateau à vapeur», et offre au public «tout ce qui peut être utile aux habitants de la campagne (...) au prix des villes». Il avise enfin sa future clientèle qu'il acceptera en paiement toutes sortes de produits, y compris grains et potasse ${ }^{18}$.

Qui dit commerce de détail à la campagne dit non seulement paiement en nature (des grains par exemple qui seront remis au marchandexpéditeur), mais également crédit: «Short-term credit was the rule made necessary by the vagaries of the seasons, the markets and personal fortunes, combined with a chronic shortage of cash.» ${ }^{19}$

Évidemment, comme le souligne lui-même Samuel Jacobs, les marchands ruraux attendent souvent un, deux ou trois ans avant d'être payés; parfois même ils ne le sont jamais ${ }^{20}$. Mais, outre la nécessité, il y a aussi des avantages à faire crédit. C'est d'abord une façon de s'attacher la clientèle. Pour les grossistes, c'est également le moyen d'assurer leurs approvisionnements en blé et autres grains. Mieux vaut, semble-t-il, souffrir des pertes «au détail», quitte à solidifier et rentabiliser ses opérations en gros.

\section{Les exportations richeloises de blé: problème de quantification}

Quelle quantité de blé un de ces négociants richelois pouvait-il exporter de la vallée annuellement? Cela, bien sûr, dépend à la fois du marchand et de l'année. Nous avons dit plus haut que les exportations de blé à Québec étaient passées d'un million de boisseaux à 22000 à peine entre les années 1802 et 1805 ; ces chiffres sont éloquents. On a prétendu, en se basant sur certains papiers de la succession Cartier, que Joseph II avait vendu assez régulièrement plus de 500000 boisseaux de blé par année à l'Angleterre, ceci au cours de la période $1815-1830^{21}$. Robert Rumilly fait état des mêmes chiffres mais il les met au crédit de

\footnotetext{
${ }^{16}$ APC, RG 11, vol. 60, liasse 84, doc. B-16, «État du commerce de la Rivière donné par W. Nelson, Esq.», s. 1., décembre 1835 .

17 A. Greer, op . cit., 285-286.

18 L'Écho du pays (Saint-Charles-sur-Richelieu), 2, 1, (6 mars 1834): 4.

19 A. Greer, op. cit., 284.

20 Ibid.

21 Henry M. Best, op. cit., 11.
} 
l'oncle, Jacques II, et non du neveu: «Jacques Cartier, grand-père de Georges-Étienne, expédiait de Saint-Jean aux États-Unis, bon an mal an, 500000 boisseaux de blé achetés dans la vallée du Richelieu.» ${ }^{22}$

Compte tenu de ce que nous savons de l'évolution du marché et de la culture du blé au XIX ${ }^{\mathrm{e}}$ siècle dans cette région ${ }^{23}$, il est bien possible que de grandes expéditions de blé aient eu lieu à l'époque de Jacques Cartier II, soit dans les années 1790-1815, mais 500000 boisseaux est un chiffre trop considérable pour un seul grossiste, même durant ces années d'abondance. En 1835, dans un mémoire faisant le point sur l'état du commerce sur la rivière Richelieu, Joseph Cartier II affirme lui-même:

Il y a eu des années ou il a sortis de la Rivière Chambly Trois Cents Mil minots de bled, mais comme ca n'est pas tout les ans le cas, l'on peut calculler à deux Cents Mil minots de bled. Quarente mil minots de Pois, Orge, Avoinne et Graine de Lin. ${ }^{24}$

Il est donc question d'un maximum de 300000 minots $^{25}$ de blé, à partager entre tous les expéditeurs du Bas-Richelieu. Si effectivement la vallée a connu certaines années à 500000 boisseaux (environ 450000 minots), il convient peut-être d'évaluer la contribution du plus important de ces exportateurs richelois à 25 ou $30 \%$ du total, soit entre 125000 et 150000 boisseaux (112 500 à 135000 minots). C'est que la basse vallée de ce premier tiers du XIX ${ }^{\mathrm{e}}$ siècle ne comptait pas moins d'une demi-douzaine de gros marchands de grains: les Cartier, Soupras, Franchère, Guérout et un ou deux autres. C'est d'ailleurs ce que nous apprend le docteur Wolfred Nelson, propriétaire de la distillerie de Saint-Denis, donc très au fait de la production et de l'exportation régionales du blé, et appelé lui aussi à dresser un bilan commercial du Bas-Richelieu ${ }^{26}$.

S'il est un peu difficile de cerner avec précision les transactions céréalières des marchands richelois des années 1790-1815, on parvient heureusement à éclairer de bien meilleure façon celles des commerçants des années 1830, grâce en particulier à ces deux brefs états commerciaux du Richelieu que nous ont légués Joseph Cartier et Wolfred Nelson. Il faut savoir, en premier lieu, que ces deux personnalités du Bas-

\footnotetext{
22 Robert Rumilly, Papineau et son temps (2 vol., Montréal, 1977), I: 410.

23 Il semble généralement accepté que l'ère du blé dans le Bas-Richelieu a couvert la période 1760-1867. L'âge d'or de cette culture intensive s'étendit de 1790 à 1815; durant les guerres de Napoléon, l'Angleterre avait grand besoin de blé. Le marché américain était également attrayant et rentable, ce qui prolongea les bonnes années du blé jusqu'en 1830. De 1831 à 1867, enfin, l'agriculture richeloise aurait été en crise. Ludger Beauregard souligne que «si l'on a pu parler d'une production de 40 boisseaux à l'acre vers 1790 , au recensement de 1851 le rendement (du blé) tombe à moins de 10.» «Les étapes de la mise en valeur agricole de la vallée du Richelieu», Cahiers de Géographie de Québec, 32 (septembre 1970): 185.

24 APC, RG 11, Vol. 60, liasse 84, doc. B-14, «Etat du Commerce de la Rivière Richelieu par Jos. Cartier, Ec., St-Antoine, 22 décembre 1835».

$25 \quad 1$ minot $=1.10^{7}$ boisseau $=39$ litres.

26 Wolfred Nelson, op. cit.
} 
Richelieu, en compagnie de messieurs Duvert, Drolet et de Saint-Ours, avaient été nommées, en 1829 , commissaires chargés de veiller à l'amélioration de la navigation sur la rivière Richelieu. C'est à ce titre que Cartier et Nelson ont rédigé leur texte respectif, en 1835.

L'intérêt de leurs déclarations réside dans le fait qu'elles abordent l'ensemble de la problématique commerciale de la région, et non seulement la question du blé. Tout d'abord la circulation des denrées sur la rivière. Cartier, dont le bilan vise, notamment, à connaître les «Droits que l'on pourrait prélever sur les vaisseaux et leur Cargaison passant dans l'Écluse» que l'on projette de construire alors dans le Bas-Richelieu, évalue à 200000 minots la quantité de blé qui est exportée chaque année par les négociants régionaux. À cela il faut ajouter, nous l'avons $\mathrm{vu}, 40000$ minots de pois, orge, avoine et graine de lin. Au chapitre des entrées dans la vallée, Cartier identifie 25000 minots de sel et 15000 minots de charbon. Au total, 280000 minots de marchandise, dans les deux sens, qui rapporteraient en droits d'éclusage $£ 291^{27}$.

Wolfred Nelson avance des chiffres assez différents. À son avis, «un marchand exporte pendant la navigation d'une année à peu près: bled: 70000 minots, orge: 8000 minots, pois: 5500 minots, avoine: 6000 minots, lin: 800 minots, faisant le tout, année moyenne, à peu près 90000 minots. Les autres marchands de la Rivière, à peu près $300000 »^{28}$. Au total, ce sont donc 390000 minots de grain qui quittent la vallée. Nelson ajoute à cela 30000 minots de sel et 20000 minots de charbon, importés dans la région, ce qui laisse voir un grand total de 440000 minots à écluser. La différence entre les chiffres des deux commissaires se situe surtout au chapitre des grains. Nelson semble avoir pris comme base de calcul des envois annuels de blé de l'ordre de 300000 minots, ce que Cartier s'est refusé à faire, considérant, nous l'avons dit, que «ça n'est pas tout les ans le cas»"

Combien de bateaux et combien de passages dans l'écluse seront nécessaires pour transporter ces milliers de minots de marchandises? Cartier, le premier, y va de ses estimations.

Daprès les connaissances que Jai du Commerce sur la Rivierre Chambly, Je suis porter à Croire qu'un Steamboat employé durant les Sept à huit mois de Navigation peut faire Cinquantes voyages de Chambly à Montréal et autant de Montréal à Chambly, ce qui ferait Cent voyages; en Admettant que ce Steamboat aurait trente Tonneaux de frette à chaque voyage, (cela) ferait Trois mil Tonneau transporter. Je pense que pour la descente des grains, que cela peut Employer Soixante Berges et autre Batiment, qui a aller et revenir feraient Cent vingt voyages. ${ }^{30}$

\footnotetext{
27 Joseph Cartier, op. cit.

8 Wolfred Nelson, op. cit

Joseph Cartier, op cit.

30 Ibid.
} 
Une écluse dans le Bas-Richelieu pourrait donc compter, selon le marchand de Saint-Antoine, sur les revenus de passage suivants:

- blé, grains, sel, charbon (280 000 minots):

- 100 voyages de vapeur, à 30 tonneaux par voyage (3 000 tonneaux):

- 100 voyages de vapeur (pour lui-même):

- 120 voyages de barges et autres bâtiments:

- 30 voyages de «bateau ouvert» chargé de madriers et bois de corde:

- 30 voyages de «bateau ouvert» (pour lui-même):

Joseph Cartier termine son bilan prospectif sur une note carrément optimiste: «Ce calcul sera Sujet à être augmenter par les années de bonne récolte, et aussitôt que le Canal de Chambly sera finis, ce qui donnera plus d'Effets à transporter.» ${ }^{31}$

Nelson, de son côté, prétend que le commerce du Bas-Richelieu peut produire, à ce moment, des revenus d'écluse de $£ 1235$. Non seulement son évaluation des quantités de marchandises à transporter dépasse-t-elle celle de Cartier, mais en plus il impose aux cargaisons et aux bateaux des droits de passage supérieurs à ceux proposés par son collègue ${ }^{32}$.

\section{Le transport des grains: sloops ou goélettes?}

Lorsque le commissaire Nelson livre ses estimations commerciales, en 1835, il indique clairement, comme Joseph Cartier d'ailleurs, que si l'on compte sur les vapeurs pour transporter le «frette» général, et sur les «bateaux ouverts» pour véhiculer madriers et bois de corde, c'est, en revanche, aux goélettes que l'on confiera les cargaisons de prix, c'est-à-dire les grains ${ }^{33}$. On range d'ordinaire dans la catégorie des goélettes, ou schooners, les voiliers à deux mâts ou plus présentant une capacité d'au moins une centaine de tonneaux. De la même façon, il est d'usage d'appeler sloops les voiliers jaugeant de 50 à 100 tonneaux. Ces petites embarcations à quille se distinguent habituellement par leur mât unique et vertical ainsi que par l'absence de pont. Si les sloops se prêtent à tous les usages, mais particulièrement à la pêche et au transport local ou régional, les goélettes, grâce au pont dont elles sont le plus souvent dotées, constituent vraiment les bateaux de prédi-

\footnotetext{
31 Ibid.

32 Wolfred Nelson, op. cit.

33 Ibid.
} 
lection des commerçants qui ont à expédier de grandes quantités de marchandises à l'abri des intempéries ${ }^{34}$.

De toute évidence, cependant, les mauvaises conditions de navigation sur le Richelieu ont amené les navigateurs et constructeurs de bateaux de la région à faire fi de ces normes maritimes. L'important pour eux était de s'adapter à la rivière, le mieux possible, et de répondre aux besoins des commerçants: distances à parcourir, effets ou denrées à transporter, en descendant comme en remontant, soins particuliers exigés par la cargaison, rapidité, etc.

Telles furent certainement les préoccupations des constructeurs de bateaux de Saint-Ours. De 1791 à 1831, ils lancèrent à cet endroit pas moins de 37 navires, dont 21 goélettes et 14 sloops $^{35}$. Notons que 31 de ces 37 bateaux avaient une capacité de 85 tonneaux et moins. En outre, si les 21 goélettes dénombrées affichaient une capacité moyenne de 64.7 tonneaux, ce qui est bien en deçà du tonnage ordinaire de ce type de navire, les 14 sloops inventoriés présentaient une jauge à peine inférieure, soit en moyenne 59.9 tonneaux. Qui plus est, cette liste de 37 bateaux montre des sloops d'environ 80 tonneaux (la Saint-Antoine et la Saint-Roch) côtoyant des goélettes de 36 (la Sainte-Claire) et de 37 tonneaux (L'hirondelle).

Cette prépondérance de la petite goélette dans les eaux du BasRichelieu témoigne du besoin bien précis des marchands de grain de la région et du souci des constructeurs de répondre à cette demande. En quoi la goélette richeloise est-elle particulière? Il s'agit d'un bateau à tonnage réduit, doté de deux mâts pour améliorer sa vitesse et sa manoeuvrabilité, et dont la cale est recouverte d'un pont afin de protéger le mieux possible les grains, cette denrée périssable et précieuse entre toutes. Pour faciliter le chargement et le déchargement, pour gagner de l'espace de cale, mais surtout semble-t-il pour restreindre le plus possible le tirant d'eau du navire, on lui aurait même dessiné une coque semblable à celles des barges. D'ailleurs, en 1835, Joseph Cartier II ne nommait-il pas ces goélettes des «berges» ${ }^{36}$ ?

34 P.-André Sévigny, Commerce et navigation sur le canal Chambly, 34-39.

35 À cette époque, Saint-Ours est l'endroit entre Montréal et Lotbinière où l'on construit le plus grand nombre de navires, exception faite de Montréal. Les statistiques de construction navale exposées ici proviennent des certificats d'enregistrement de navires émis au port de Québec à partir de 1787 [APC, RG 42, A1, Quebec shipping Registers, vol. 183-198]. Pierre Dufour, de Parcs Canada, a rassemblé et compilé les certificats du port de Québec pour les années 1787 à 1900 dans le cadre d'un travail de recherche sur la construction navale à Québec aux $18^{\mathrm{e}}$ et $19^{\mathrm{e}}$ siècles.

36 Cela est d'autant plus plausible que, encouragée pourrait-on dire par l'aménagement des canaux canadiens et américains dès les années 1820, la construction de ces barges au profil si caractéristique (fond plutôt plat, flancs presque verticaux) avait sûrement capté l'attention des marchands et navigateurs richelois. D'ailleurs entre 1823 et 1833 sur le lac Champlain, on comptait pas moins de 232 barges-voiliers. Ralph N. Hill, Yankee Kingdom: Vermont and New Hampshire (New York, 1973), 171. 
Si ces goélettes étaient destinées à arpenter le fleuve, au moins jusqu'à Québec, les sloops «tout usage» se consacraient surtout au cabotage régional: Montréal, lac Saint-Pierre, Mauricie et, bien sûr, Bas-Richelieu. Leur gréement, surtout l'absence de pont, leur interdisait habituellement les trop longs voyages. Le 25 mai 1829, Matthew Bell, le directeur des Forges du Saint-Maurice, profitait du passage à Trois-Rivières du sloop Godefroy, dont le capitaine était Pierre Cormier de Saint-Ours, pour faire parvenir au marchand Eustache Soupras, de Saint-Mathias, une commande de barres de fer, socs de charrue, marmites, chaudrons, fers à cheval et poêles. L'occasion était belle puisque le capitaine Cormier se rendait justement livrer du sel et autres produits au marchand Franchère, aussi de Saint-Mathias ${ }^{37}$.

En l'absence de goélettes, il arrivait fréquemment que des sloops soient appelés à être chargés de grains. Parfois, également, une commande réduite de blé ne justifiait pas l'utilisation d'une goélette. Enfin, l'urgence d'une livraison venait souvent s'ajouter à ces contraintes. Un des directeurs des Forges de Batiscan écrivait en 1810 au marchand Pierre Guérout de Saint-Denis:

Nous trouvant actuellement dans un grand besoin de farrine, je vous prie de faire toute diligence pour embarquer à bord du premier Bâtiment qui se présentera, les 500 minots de Bled ainsi que les pois que Mr. [Thomas] Coffin a acheter chez vous. ${ }^{38}$

Pour être assuré d'une livraison, même par l'entremise d'un sloop, il fallait habituellement grossir la commande:

Faites nous le plaisir de trouver quelque occasion prompte pour nous envoyer les 100 Minots de Bled que vous avez; et comme cette quantité est si inconsidérable pour une charge de Bâtiment, nous prendrons 300 à 400 Minots de plus. Ayez la bonté de nous écrire quels seront vos termes pour cette quantité additionnelle, payable en fonte dans le courant de Septembre. ${ }^{39}$

Il appert donc qu'à cette époque, c'est-à-dire dans les années 18101812, la commande minimale de blé se situait autour de 400 à 500 minots. À remarquer aussi que le fournisseur de blé, Pierre Guérout, sera payé en nature par les directeurs des Forges de Batiscan.

Tous les grossistes en grains du Bas-Richelieu en vinrent à posséder au moins une goélette. En 1825, les Cartier de Saint-Antoine en avaient deux, une construite à Saint-Ours et l'autre, l'Abeona, lancée à

\footnotetext{
37 APC, MG23, GIII, 25 (Collection of the Antiquarian and Numismatic Society of Montréal), Series 1, doc. 819, Three Rivers, 25 May 1829.

38 Parcs Canada, Québec (ci-après PCQ), Histoire et Archéologie, Cahier de correspondance des Forges de Batiscan (ci-après $\mathrm{CFB}$ ), B.J. Frobisher à Pierre Guérout, Batiscan, 6 août $1810,299$.

PCQ, CFB, B.J. Frobisher à Pierre Guérout, Batiscan, 27 avril 1812, 407.
} 
Chambly et jaugeant 118 tonneaux ${ }^{40}$. Mais au début du siècle, les bateaux pourvus d'un pont étaient, de toute évidence, beaucoup moins répandus. Les grains d'exportation, lorsqu'ils n'étaient pas déjà tachés à l'embarquement, c'est-à-dire cariés par un champignon parasite, risquaient fort d'arriver à Québec en piteux état à cause d'un transport à ciel ouvert. Dans un cas comme dans l'autre, les agents des expéditeurs, à cet endroit, n'avaient souvent d'autre choix que de «faire battre un banc au Marché, pour inviter les habitants de venir acheté du Bled de Semence» ${ }^{41}$.

S'il n'était pas facile d'écouler du blé taché, cela n'empêchait pas les grossistes d'y aller de quelques tours de passe-passe pour y parvenir. En octobre 1801, par exemple, Jacques Cartier II était à Québec où il venait de recevoir de Saint-Antoine-sur-Richelieu un chargement de blé rance et d'apparence médiocre. En prévision d'un nouvel arrivage, l'oncle transmit alors ses instructions à son neveu Joseph, pour le cas où le blé prêt à être embarqué aurait été partiellement gâté:

À l'égard du Bled taché, il faut faire comme cecy. Premièrement, envoyer le beau bled le premier; après cela le faire pelté dans le batiment dans les deux bcuts; ensuite envoyer le bled taché pour le mettre dans le centre du panneaux; ça fera qu'en deschargeant ici, il sera enlevé le premier. ${ }^{42}$

Afin donc de préserver le blé d'une dégradation supplémentaire (qui lui aurait probablement enlevé toute valeur marchande), les expéditeurs du Richelieu se munirent à peu près tous d'au moins une goélette à grain, dans les années 1820 et 1830 . Bien évidemment, toutefois, un ou deux de ces bateaux ne parvenaient pas à effectuer toutes les livraisons d'un marchand important; celui-ci devait par conséquent soit affréter quelques embarcations en sus pour la saison de navigation ${ }^{43}$, soit louer les services d'un capitaine et de son bateau «au voyage»: "Vous nous annoncez avoir engagé Cormier qui doit nous apporter 500 Minots de Bled et prendre vos effets. ${ }^{44}$

Mais ces navires d'appoint étaient presque toujours des sloops et plusieurs parmi eux, en raison de l'âge surtout, n'étaient pas étanches. En 1811, Thomas Coffin n'était pas satisfait de la qualité du blé reçu à Batiscan et il s'en plaignit à son fournisseur, Pierre Guérout: «J'observe ce que vous dites au sujet du Bled. Je puis vous assurer qu'il était humide au point de ne pouvoir être moulu. La diminution lorsqu'il sera

\footnotetext{
$40 \quad$ Brian Young, op. cit., 145.

41 ANQQ, Petites Collections, AP-P-334 (fonds Jacques Cartier), Blackwood, Paterson et Co. à Jacques Cartier, Québec, 3 mai 1804.

42 Ibid., AP-P-335 (fonds Joseph Cartier), Jacques Cartier à Joseph Cartier, Québec, 19 octobre 1801 .

43 PCQ, CFB, Thomas Coffin à Pierre Guérout, Batiscan, 11 septembre 1809, 252.

44 Ibid., 7 octobre $1811,378$.
} 
sec doit être grande.» ${ }^{45}$ Est-il besoin de dire que les clients insistèrent de plus en plus, et à l'avance, pour qu'on leur livre du blé qui «sera beau et d'une bonne qualité, point taché» ${ }^{46}$. On alla jusqu'à demander que la livraison soit faite par goélette; plus encore, on insista même pour retenir le capitaine et l'embarcation de son choix ${ }^{47}$.

En pleine saison de navigation, cependant, particulièrement au printemps et à l'automne, les bateaux disponibles se faisaient rares et l'acheteur se comptait déjà chanceux si sa marchandise lui était livrée. On voyait des marchands expédier des effets à un client avant même que ce dernier n'ait eu le temps de confirmer par écrit une commande verbale, ceci par «crainte de ne point voir s'offrir d'autre Batiment pour vos endroits» ${ }^{48}$. Parfois même, les négociants conseillaient de réserver un bateau quelques mois à l'avance si l'on désirait s'assurer d'une livraison ${ }^{49}$. Mais les capitaines du temps se savaient peu nombreux et indispensables. Faut-il se surprendre qu'ils aient voulu en tirer un bon parti? «Je viens d'engager la goélette de Pierre Bigué pour descendre le Bled, Pois et Avoine. Il a fallu lui promettre 1600 Minots et je vous prie (...) de complété sa charge avec des Pois et de l'avoine..> ${ }^{50}$

\section{Conclusion}

Durant le premier tiers du dix-neuvième siècle, le Bas-Richelieu, grâce à un sol et à un climat exceptionnellement favorables, s'est imposé comme le grenier du Bas-Canada. La culture et le commerce des grains, et en particulier du blé, firent la prospérité de la basse vallée. Mais il est clair, bien que la chose soit rarement mentionnée, que la navigabilité déficiente de la rivière a restreint cette bonne fortune en gênant considérablement la mise en marché des céréales de la région.

Pour s'en convaincre, ne suffit-il pas de savoir qu'à l'été de 1811, par exemple, les eaux du Richelieu sont tellement basses que les marchands Thomas Coffin, de Batiscan, et Pierre Guérout, de Saint-Denis, ne peuvent commercer ensemble? Ils n'ont d'autres choix que d'affréter deux «chaloupes du Haut-Canada qui portent environ 10 tonneaux (...), vont à la Rame, à la perche et dans les rapides ainsi que dans les courants, et à la cordelle (...), ne tirent qu'un pied d'eau» ${ }^{51}$.

Été exceptionnel? Tout au contraire. Chaque année, à la même période, la situation se répétait. Évidemment, les «chaloupes du Haut-

\footnotetext{
45 Ibid., 23 juin $1811,340$.

46 Ibid., 11 avril $1811,320$.

47 Ibid., 21 septembre 1811, 368; 12 avril 1811, 319; 4 novembre 1811, 385.

48 Ibid., B.J. Frobisher à André Winklefoss, Batiscan, 29 septembre 1808, 154.

49 Ibid., Thomas Coffin à Pierre Guérout, Batiscan, 11 septembre 1809, 252.

so Ibid., 12 avril 1811,321 .

51 Ibid., 18 juillet $1811,346$.
} 
Canada ${ }^{52}$ ne seront guère utilisées par les marchands richelois pour livrer les céréales. La faible capacité de ces embarcations amènera plutôt les expéditeurs à opter pour le transport par goélette. Mais cette méthode nécessitait un transbordement de cargaison à Saint-Ours, étape dont le coût n'était pas le moindre embarras. En 1834, les marchands de grain de Saint-Charles déboursaient de trois à quatre sols pour chaque minot de céréale transporté en barque depuis leur village jusqu'à Saint-Ours: «C'est plus de moitié de ce qu'il en coûte pour faire transporter ces produits d'ici à Québec lorsque les goélettes peuvent charger ici», se plaignait alors le rédacteur du journal local ${ }^{53}$.

Ce n'est qu'en 1849 que l'écluse et le barrage Saint-Ours vinrent stimuler la navigation commerciale dans le Bas-Richelieu. Les bateaux purent alors compter sur une profondeur d'au moins $2.1 \mathrm{~m}$ jusqu'au bassin de Chambly. Pour le blé, hélas, ces améliorations survenaient vingt ans trop tard. Ce n'était pourtant pas la faute des Cartier, Nelson, Franchère, Soupras et autres hommes d'affaires de la région qui avaient compris depuis belle lurette que le commerce des céréales reposait sur trois piliers et non deux: l'offre, la demande et la libre navigation de la rivière.

\footnotetext{
52 Il semble s'agir des fameux Durham boats, ces bateaux américains qui firent leur apparition sur le haut Saint-Laurent en 1809. Robert Passfield, «Ordnance Supply Problems in the Canadas: The Quest for an Improved Military Transport System, 1814-1828», Journal for the History of Canadian Science, Technology and Medecine, 5 (septembre 1981): 192.

L'Écho du pays (Saint-Charles-sur-Richelieu), 2 octobre 1834, 3.
} 